\title{
Programming Strategies Using an Actor-Based Environment
}

\author{
Raul Sidnei Wazlawick and Antonio Carlos Mariani \\ UFSC-CTC-INE \\ Florianópolis, SC - Brazil \\ \{raul,mariani\}@inf.ufsc.br
}

\begin{abstract}
This text describes some programming strategies that computer science students have used to conduct programming tasks with an actor/stage metaphor. The authors maintain that the actor/stage metaphor is a useful strategy for teaching programming skills in the early classes of a computer science or engineering program, especially if a concurrent teaching strategy is used. The evidence of the success of this approach is that students in the first semester of a computer science course were able to develop many kinds of simulation software and games. This article shows some strategies that students have used, and the underlying concepts behind their approaches, and discusses the utility of the actor/stage metaphor as an educational tool.
\end{abstract}

\section{INTRODUCTION}

Programming languages are usually taught in computer science and engineering courses through a very strict bottom-up and sequential approach. However, Bertrand Meyer [1] has proposed to invert the Computer Science curriculum. However, any such attempt must rely on good methodologies and must be supported by high quality tools. Silveira and Scavarda-doCarmo [2] have also discussed different techniques for teaching engineering, and proposed a mix of sequential and concurrent teaching, based on active learning and learning-by-doing for teaching engineering. 
This paper describes some results of a five-year experience where a new approach to teaching programming has been tested. The methodology consists in asking first-year students to produce solutions to problems that seem complex for beginners, but that can be solved if a suitable metaphor is adopted.

It was found that the projects produced by students who use an actor/stage metaphor are far more complex and well structured than projects produced by students submitted to traditional teaching approaches.

\section{BACKGROUND AND RELATED WORK}

The tool "Actors' World", developed to support this kind of teaching method, was inspired initially by the Logo language and environment developed in the 1960's [3]. A second source of inspiration was Swarm [4], a software package for multi-agent simulation of complex systems. The main characteristic of Swarm that was incorporated by Actors' World was the implementation of an open architecture where different execution models could be implemented. The current version implements only the discrete events model, which is similar to that used in Swarm to describe agents' behavior. The main difference is in the notation used to describe behavior, which is similar in some aspects to that used in the Ágora environment [5].

The word "actor" in this environment is not intended to be consistent with that proposed by researchers such as Agha [6]. The concepts of stage and actors were chosen only to offer a metaphor to a clear and consistent interpretation in the world.

\section{BASIC CONCEPTS}

The programming tool Actors' World was developed in 1996 using the Smalltalk/V environment. It incorporates two major concepts: Actor and Stage.

Actors are instances of the Actor class. Actors behave like the turtle in the Logo language in the sense that they have a graphic representation, a position on the stage and a heading direction. Actors can move forward (method "go:") and turn around (method "turn:"). Actors can also sense if other actors are touching them, if a different actor is in the vicinity and so on. Each actor has a role that is specified by a set of sentences in the method "defineRole". Five basic kinds of sentences are allowed:

a) Unconditional action: defined by the method "alwaysDo:". For example, self alwaysDo: [self go: 5; turn: 3 ] 
b) Conditional action: defined by the method "when:do:". For example, self when: [self isBeingTouched] do: [self go: 10]

c) Fixed-interval actions: defined by the method "each:do:". For example, self each: 30 do: [self go: 10]

d) Probabilistic actions: defined by the method "withProbabilityOf:do:". For example: self withProbabilityof: 0.1 do: [self turn: 30]

e) Specific time actions: defined by the method "at:do:". For example: self at: 1000 do: [self leavestage]

The stage is the environment where actors live and interact. Each actor keeps a reference to the stage in which it is included, and the stage knows which actors it contains. The stage also controls the execution of the actors' roles, using a mechanism known as discrete events parallelism. Discrete events parallelism permits each actor to execute every unconditional sentence and every other kind of sentence that is supposed to be executed at that discrete instant of time without interruption. This is not real parallelism because there is not a real time-slice for each object. Using this approach, many problems related to object concurrency do not appear. For instance, debugging is still possible in Smalltalk, because sentences are executed orderly in discrete time. People trying to debug a real parallel Smalltalk program usually have a difficult time. One disadvantage of discrete event parallelism is that one single object can take over the processor by entering into a loop, and disabling other objects from running. But if a programming style with simple sentences is used instead of long and complex ones, then these problems usually do not occur.

\section{STUDENTS' STRATEGIES IN ACTOR BASED PROGRAMMING}

The course of object-oriented programming in the first year of the Computer Science career is based on a methodology similar to that proposed by Silveira and Scavarda-do-Carmo [2], where concepts are not viewed from bottom-up, but in parallel, and in a problem-driven way. Basic programming tools are presented to the students, who are then asked to develop applications. After working on some collective projects such as a traffic light system [7], they are asked to propose new projects. The teacher has to evaluate if the proposed projects are suitable in size and complexity for the student to be exposed to the main concepts of object oriented programming.

One group decided to implement a game based on "Space Invaders". The game has a mother ship that launches smaller ships. Each smaller ship launches bombs that fall to the ground, where a gun controlled by the user is located. The user is able to move the gun left and right, and fire missiles. A 
single missile can destroy a bomb or a small ship, but a lot of missiles are necessary to destroy the mother ship. It is evident that developing such a game is quite a challenge for a first year student, but using the expressive power of the actor based environment allowed this challenge to be accomplished relatively easily.

The solution involved the creation of the following actor classes: Gun, ExplodedGun, MotherShip, ExplodedMotherShip, Missile, Bomb, ExplodedBomb, ExplodedMissile, Ship, and ExplodedShip. The occurrence of these objects as graphic entities in the proposed problem helped the students to identify them correctly. Different behaviors between missile and exploded missiles, for example, were used to identify subclasses.

The Gun class has the following behavior: when it is off the stage, it comes back to the stage (the user cannot place the gun outside the stage), and when it is touched by an instance of an ExplodedBomb or an ExplodedShip, it becomes an ExplodedGun:

defineRole "in class Gun"

self when: [self outofstage ] do: [self turn: 180; go: 35]. self when: [self touchesActorofType: ExplodedBomb] do: [self become: ExplodedGun] .

self when: [self touchesActorOfType: ExplodedShip] do: [self become: ExplodedGun] .

An ExplodedGun and its counterparts have very simple behavior. Once created they have to exist for a short time and then disappear:

defineRole "in class ExplodedGun"

self at: 10 do: [self leavestage].

Soon the student realizes that exploded things always have the same behavior, and that the existence of different classes of exploded things is not necessary, because the only difference is their image (bitmap) shown on the screen. At this point, it is a good idea to discuss the question of when it is interesting to have different subclasses or only a single class with different attributes.

The MotherShip behaves erratically. From time to time it jumps to a different region of the stage. Also, from time to time it launches a ship. When it touches any object it decreases its shields. When shields reach the value 0 it becomes an ExplodedMotherShip:

defineRole "in class MotherShip"

self each: 40 do: [self jump]. "jump is implemented elsewhere"

self each: 15 do: [self launchShip]. "launchship if defined below"

self when: [self touchanActor] do: [live := live - 1].

self when: [live $=0$ ] do: [self become: ExplodedMotherShip].

This kind of situation is useful for introducing the concept of instance variable. Also, method decomposition is explored at this stage because the 
student sees that it is simpler to read a method when it is decomposed in meaningful parts. The implementation of the method "launchShip" is:

launchShip "in class MotherShip"

| ship |

ship := Ship new.

ship enterStage: self stage. "the ship enters the same stage as its mother ship"

ship jumpto: self position. "the ship goes to the position of the mother ship"

The definition of the other classes follows more or less the same pattern. A ship still has interesting behavior. It has to move around the stage, drop bombs, feel if it has touched the ground or an exploded missile and in this case becomes an ExplodedShip:

defineRole "in class Ship"

self alwaysDo: [self go: 4].

self withProbabilityof: 0.2 do: [self dropBomb].

"the implementation of dropBomb is very similar to launchship"

self when: [self hitGround] do: [self become: ExplodedShip].

"hitground is simply a position test"

self when: [self touchActorofType: ExplodedMissile] do: [self become: ExplodedShip].

This project was successful and the final result was a fully playable game.

\section{CONCLUSIONS}

This experience has been remarkably surprising and gratifying. After using this approach with more than 300 students it was evident in each project developed that the underlying concepts of object-oriented programming were adequately learned from scratch, or reorganized when students already had misconceptions about object-oriented programming. Students proved that they were able to understand the concepts of objectoriented programming through this actor-based extension, and even when they implement their first system incorrectly, they were able to see better approaches. Some major difficulties with the syntax of the Smalltalk language are addressed in [7].

This approach to teaching programming is not limited to one semester. The method suggested is to start the course (first semester) with this actorbased extension to Smalltalk, so that students may learn the major concepts of object-oriented software analysis and synthesis. In the second semester it is suggested that students work with a typed language such as Eiffel, where types, exception handling, programming by contract, multiple inheritance 
and other concepts may be presented to students that already have an idea of how to model a system. In the third semester it is suggested that students work with a medium level language, such as $\mathrm{C}++$, where a traditional algorithmic approach may be presented. This third semester discipline may be combined with a course on data structures, where the student is supposed to develop classic algorithmic thinking.

As a continuation of this work a set of design patterns for student constructions in the Actors' World is under development. The catalog of design patterns is intended to help students in the future learn how to solve common programming tasks using this tool. Examples of common patterns are actors that reproduce themselves or that produce actors of different types, actors that react to touch, actors that react to the presence of other actors within a given range, and so on.

\section{REFERENCES}

[1] B. Meyer "Towards an Object-Oriented Curriculum" Symposium on Teaching Object Technology at TOOLS 11 (Technology of Object-Oriented Languages and Systems), Santa Barbara, August 1993; Prentice Hall, 1993, pages 585-594.

[2] M. A. da Silveira and L. C. Scavarda-do-Carmo, "Sequential and Concurrent Teaching: Structuring Hands-On Methodology", IEEE Transactions on Education, Vol. 42, No. 2, May 1999.

[3] S. Papert, "Mindstorms: Children, Computers and Powerful Ideas," New York. Basic Books, 1980.

[4] N. Minar, R. Burkhart, C. Langton, M. Askenazi, "The Swarm Simulation System: A Toolkit for Building Multi-agent Simulations," Santa $\mathrm{Fe}$ Institute, http://www.santafe.edu/projects/swarm/overview/overview.html, 1998.

[5] M. Q. Marchini and L. F. B. Melgarejo, "Ágora: Groupware Metaphors in ObjectOriented Concurrent Programming," in ECOOP 94 Workshop on Models and Languages for Coordination of Parallelism and Distribution, 1994.

[6] G. A. Agha, Actors: A Model of Concurrent Computation in Distributed Systems. Cambridge: MIT Press, 1986.

[7] R. S. Wazlawick and A. C. Mariani, "The Use of an Actor and Stage Metaphor for Introducing Object-Oriented Programming," IFIP World Computer Congress, 2000. 